\title{
Plasticity of Myelinating Glia
}

Until recently, myelin has been considered a passive structural component of neural circuits, necessary for fast action potential propagation but not contributing in a dynamic way to nervous function. The possibility that myelin or myelinating cells might contribute to neural plasticity, other than in myelin repair, was not seriously considered. Indeed, the idea was strongly resisted or rejected outright by many experts. This conceptual barrier is now being dismantled by recent research that reveals plasticity of myelinating glia in a number of different contexts. This Special Issue explores this new and growing area of neuroscience through a collection of invited articles from some of the leaders in the field. Naturally, the selection of authors is far from comprehensive because of the constraint of physical space and our own limitations of imagination and persuasion as guest editors.

Oligodendrocytes were the last major type of glial cell to be recognized, and their anatomy and function were initially mysterious. In contrast to astrocytes and microglia, some primary functions of which could be deduced by histology - showing, for example, astrocytes bridging between blood vessels and neurons, or microglia surrounding sites of injury - Schwann cell and oligodendrocyte functions were enigmatic. The slender cellular extensions from the oligodendrocyte cell body to axons could not be resolved by histological stains and microscopy in the era when Ramon y Cajal was feverously identifying cells in the brain and deducing their functions, so there was nothing to associate oligodendrocytes to axons anatomically or physiologically. Although Schwann cells were clearly evident as cells strung sequentially along axons in the peripheral nervous system, their function could not be deduced from their anatomy alone. Nothing suggested that Schwann cells and oligodendrocytes might be analogous to one another in any way.

The main impediment to understanding both of these cell types was that neither the structure nor the function of myelin was understood. Even into the mid-twentieth century, myelin was regarded as a fatty substance that was extruded from axons. This substance coated some but not all axons (and none in invertebrate animals), leading 
Cajal to reject the proposal that myelin provided a vital function as electrical insulation, required for transmission of electrical impulses. Moreover, Schwann cells were considered by early anatomists to be vestigial cells that had formed the nerve axon during development by splicing together axonal segments into a continuous tube.

Del Rio Hortega, an associate of Cajal's, perfected a method that stained the slender processes from oligodendrocytes, providing the critical missing evidence linking these cells to axons, an observation that was first confirmed by famed neurosurgeon Wilder Penfield in 1924. Ichiji Tasaki, through elegant single fibre electrophysiological studies, determined that action potentials were transmitted along myelinated fibres in a saltatory fashion, jumping rapidly from node to node. Electron microscopy in the 1950's revealed the completely unexpected finding that the presumed fatty exudate from axons, myelin, was formed by a highly compacted series of membrane wrappings around the axon by Schwann cells in the PNS and oligodendrocytes in the CNS, an intricate intercellular junction seen in no other cellular context.

Advances in imaging technology have been crucial for understanding the structure and plasticity of myelin and myelinating glia. This continues today both from advances in in vivo cellular imaging in live animals and from MRI brain imaging methods, such as diffusion tensor imaging, that resolve white matter microstructure and plasticity in the human brain. In this Special Issue, Hill and Grutzendler survey advances in live in vivo cellular imaging, including new fluorescence and label-free microscopic imaging methods, and describe how these new technologies are being used to illuminate the morphological plasticity of oligodendrocytes during myelin formation, remodeling, damage, and repair. Bells et al. review novel MRI brain imaging approaches that provide more specific information about white matter microstructure and plasticity in the human brain, in relation to cognitive development from childhood through adulthood. The intriguing idea that myelin plasticity improves information processing by optimizing synchrony of spike time arrival is addressed experimentally in their paper by assessing neural phase synchrony in visual cortex during a visual task in patients with recurrent 
demyelinating syndromes, compared to age-matched controls. This has revealed a correlation between white matter microstructure and neural synchronization in cognitive processing.

Experimental evidence is accumulating from work with mice and zebrafish that electrical activity in axons can modulate myelin production and morphology at several levels, including the control of oligodendrocyte development and long-term survival, the rate of synthesis and assembly of myelin structural proteins, the length, number and thickness of myelin internodes elaborated by individual oligodendrocytes, and the microarchitecture of nodes of Ranvier and the paranodal regions. These features of myelin have the potential to influence impulse conduction speed as well as other "non-canonical" functions of oligodendrocytes such as metabolic coupling between oligodendrocytes and their associated axons, or increasing the maximum firing rate of the neuron-oligodendrocyte unit. Two articles in this issue by Foster, Bujalka and Emery and by Daumante, Lyons and Livesey deal with some of these concepts and mechanisms - collectively referred to as "adaptive myelination" - and the potential consequences for neural circuit function and animal behaviour. In contrast to long axons that project over great distances in white matter tracts, where spike time latencies are substantial, many interneurons in the grey matter of the cerebral cortex and elsewhere have relatively short axons. That some of these short axons are myelinated suggests that myelin in grey matter might fulfil a noncanonical function such as those referred to above. Timmler and Simons focus their review on the role of this "grey matter myelin", discussing its potential role in supporting axons metabolically, inhibiting axon sprouting (thereby constraining neural circuit connections and plasticity), promoting synchrony of spike time arrival (permitting signal summation at the target) and/or in tuning the frequency, phase and coherence of neural oscillations.

The appreciation that neural activity influences the formation and remodeling of myelin opens further paths of investigation into how action potential activity is communicated to glia (and other cells) situated along the axon, far from both synapses and the neuronal cell body. Neurotransmitters are ideally suited for activity-dependent axon-glial communication and this could explain why myelinating glia express many different types of 
neurotransmitter receptors. The surprising discovery that physical synapses are formed on at least some oligodendrocytes and their precursors provides an obvious mechanism for how neural impulse activity could influence the development of myelinating glia and/or the synthesis of myelin sheaths. However, it is not clear whether synapses are present on all oligodendrocyte lineage cells and, so far, there is no evidence that Schwann cells participate in synapse formation. Volume conduction - that is, release of neurotransmitter by vesicular and non-vesicular release mechanisms outside of synapses - is also used to communicate electrical activity from axons to myelinating glia and such non-synaptic signalling appears well suited to influence biological responses in myelinating glia, which might not require stimulation on the millisecond time scale that synaptic transmission provides. Two articles in this issue, by Kula, Chan and Kukley and by Habermacher, Angulo and Bessamer, are concerned with activity-dependent signaling between axons and oligodendrocyte lineage cells. Kula et al. discuss glutamate receptor function in oligodendrocyte precursors (OPCs) and oligodendrocytes, with a special focus on differences between synaptic and non-synaptic signaling. They survey the expression of different classes of glutamate receptors (AMPA, NMDA, mGluR, and Kainate) in oligodendrocyte lineage cells and review what we know about the functional roles of this mode of signalling. Habermacher et al. also discuss synaptic versus non-synaptic axon-oligodendrocyte signaling by glutamate and GABA - for example, the release of glutamate at axon varicosities, as has also been shown for the axo-glial neurotransmitter ATP.

Acetylcholine receptors and acetylcholinesterase (AChE) enzymes are present in oligodendrocytes, raising the question of how cholinergic signaling might affect oligodendrocyte development, function, and myelination. A study reported by Belgrad, Dutta et al. approaches this question from the unusual perspective of Gulf War Illness (GWI). This illness, characterized by a wide range of cognitive and neurological disabilities, afflicted members of armed services returning from the first Gulf War (1990-91). Over twenty-five years later many veterans remain affected, yet the cause of GWI is still unknown. A leading theory, supported by epidemiological data, is that low-level exposure to Sarin nerve agents, which act as AChE antagonists, might be an underlying cause. Research on the role of AChE inhibitors in GWI has focused on their adverse effects on neurons, but 
Belgrad, Dutta et al. report that they also detrimentally affect oligodendrocyte development and survival, potentially implicating oligodendrocytes in the pathophysiology of GWI.

In addition to interactions with axons, the interactions of oligodendrocytes with other brain cells is important for normal function and plasticity in the nervous system. Microglial involvement in demyelinating disorders is well established but, as outlined in the review article by Bar and Barak, a role for microglia in normal myelin development and physiology is also starting to emerge. The release of cytokines, growth factors and proteases, as well as tissue remodeling by microglia implicates microglia-myelin interactions in several neuropsychiatric and neurodevelopmental disorders including autism spectrum disorder, schizophrenia and age-related cognitive decline.

Some types of practice-intensive learning, notably motor skills learning, is "consolidated" during post-training sleep; that is, performance of the skill in question is better immediately after a period of sleep than at any time leading up to it. This implies that training initiates some longer-term process, possibly involving changes at the level of brain cells, that continues during sleep. In this issue, Bellesi describes work on the circadian nature of oligodendrocyte development, showing that OPC proliferation is more likely to occur during a mouse's daytime inactive period and differentiation during the night-time active period, tentatively suggesting a link between learning consolidation and the cellular dynamics of oligodendrocyte development.

Extracellular signals, including activity-dependent signals, impinge on the transcriptional program of the receiving cell (e.g. OPCs or Schwann cells) by modulating the transcriptional machinery - that is, DNA-binding transcription factors in concert with chromatin remodeling complexes. This complex interplay, played out in the nuclei of oligodendrocyte lineage cells or Schwann cells, is what ultimately controls myelin dynamics during development or regeneration. This central topic of transcriptional control of myelination in the CNS and PNS is reviewed in this issue by Sock and Wegner. One specific example of a gene product (a long non-coding RNA) that is transiently up-regulated as murine OPCs differentiate into pre-myelinating oligodendrocytes is described 
by Kasuga, Fudge and Li. This and other developmental stage-specific gene products will be invaluable for studying the oligodendrocyte dynamics during early postnatal development, during adaptive myelination in adults and, potentially, during remyelination during demyelinating diseases like multiple sclerosis.

Demyelination and subsequent remyelination is thought to be a common occurrence during other types of brain insult, not only multiple sclerosis - during stroke or acute spinal injury, for example. In this vein, McTigue et al. review what is known about the role of white matter sparing and post-injury repair during recovery from spinal cord injury, whether remyelination is critical for functional recovery and the factors (age, physical exercise, the microbiome, therapeutic drugs ... ) that might influence the recovery response.

Regeneration in the peripheral nervous system (PNS) - following a nerve crush or transection, for example - is the topic of the final article by Stierli, Imperatore and Lloyd. They describe fascinating differences between the modes of production of remyelinating cells in the PNS versus the CNS (notably the lack of a resident precursor cell population in peripheral nerves), and the remarkable post-injury plasticity of Schwann cells that allows them to exit the nerve and contribute to regeneration of surrounding non-nervous tissue.

We hope you the readers enjoy and draw inspiration from this special collection of articles. We thank all the contributors for illustrating just how much our ideas about myelinating cells have evolved over recent years, from structurally intriguing but functionally static bystanders to active, busy participants in nervous system function.

\section{Douglas Fields $^{1}$ and William D Richardson ${ }^{2}$}

${ }^{1}$ Section on Nervous System Development and Plasticity, The Eunice Kennedy Shriver National Institute of Child Health and Human Development, National Institutes of Health, Bethesda, MD 20892, USA.

${ }^{2}$ Wolfson Institute for Biomedical Research, University College London, Gower Street, London WC1E 6BT 